\title{
Beïda Chikhi, Assia Djebar. Histoires et fantaisies
}

\author{
Ilaria Vitali
}

\section{(2) OpenEdition}

\section{Journals}

\section{Edizione digitale}

URL: http://journals.openedition.org/studifrancesi/9057

DOI: 10.4000/studifrancesi.9057

ISSN: 2427-5856

\section{Editore}

Rosenberg \& Sellier

\section{Edizione cartacea}

Data di pubblicazione: 1 octobre 2008

Paginazione: 498-499

ISSN: 0039-2944

\section{Notizia bibliografica digitale}

|laria Vitali, «Beïda Chikhi, Assia Djebar. Histoires et fantaisies», Studi Francesi [Online], 155 (LII | II) | 2008, online dal 30 novembre 2015, consultato il 12 janvier 2021. URL: http://journals.openedition.org/ studifrancesi/9057 ; DOI: https://doi.org/10.4000/studifrancesi.9057

Questo documento è stato generato automaticamente il 12 janvier 2021.

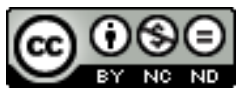

Studi Francesi è distribuita con Licenza Creative Commons Attribuzione - Non commerciale - Non opere derivate 4.0 Internazionale. 


\title{
Beïda Chikhi, Assia Djebar. Histoires et fantaisies
}

\author{
Ilaria Vitali
}

\section{NOTIZIA}

BË̈DA CHIKHI, Assia Djebar. Histoires et fantaisies, Paris, Presses Universitaires de Paris Sorbonne, 2007 («Lettres Francophones»), pp. 197.

1 Assia Djebar. Histoires et fantaisies è un saggio che chi ama la scrittrice maghrebina non potrà fare a meno di inserire nella propria biblioteca. Da anni, Beïda Chikhi, specialista di letteratura francofona e in particolare maghrebina, docente all'Université Paris IVSorbonne e direttrice del Centre International d'Etudes Francophones, ha abituato il lettore alla precisione, chiarezza ed intelligenza della sua analisi critica. Il suo viaggio nella geografia dell'imponente opera djebariana è incominciato negli anni Ottanta, con il saggio Les Romans d'Assia Djebar, edito da OPU nel 1989 (e di cui è stata pubblicata nel 2002 una versione «augmentée et refondue»), il secondo testo critico dedicato all'opera di Djebar dopo quello di Jean Déjeux. In questo nuovo saggio, edito dalle Presses de Paris Sorbonne, l'autrice sceglie di apportare «le complément d'expérience et de vécu, qui s'est accumulé au fil du temps, du côté de la grande romancière comme du côté de la lectrice et de l'universitaire que je suis» (p. 7). È un saggio che non nasconde l'entusiasmo nel parlare di una delle più grandi voci del nostro tempo, autrice di un'opera che si fa edificio imponente, pietra miliare, punto di riferimento incontournable. Un'analisi, quella di Chikhi, che, come spiega nell'introduzione, non vuole essere soltanto storica o stilistica, ma che intende toccare «les fantaisies», la sensibilità dell'essenza poetica, l'ispirazione pittorica e musicale, l'indagine del segreto dell'essere che rendono unica la prosa di Assia Djebar.

2 A partire dal primo romanzo, La Soif, definito dall'autrice «un air de flûte», si sviluppa la melodia di un'opera maestosa, qui orchestrata e analizzata in quattro parti. Nella prima, «L'histoire au cœur du romanesque», Chikhi entra subito nel vivo della 
produzione di Assia Djebar, soffermandosi sull'importanza della Storia e della storia nell'opera della scrittrice. Molti dei suoi romanzi fanno leva sulla memoria, dal recupero di una tradizione araba problematica fino al racconto degli anni più tragici $\mathrm{e}$ al (bi)sogno di un'Algeria «vive et tatouée». Un viaggio in un passato drammatico in cui le donne svolgono un ruolo di primo piano: «Au cours de ces années tragiques les femmes ont, une fois de plus, animé intensément la scène historique et médiatique, clamant fort leur refus de mourir. En lisant les œuvres posthumes de Taous Amrouche, les dernières publications d'Assia Djebar, de Malika Mokeddem, de Leïla Sebbar, de Nina Bouraoui, et de quelques autres d'autres romancières, on penserait volontiers que les fictions s'engagent à nouveau dans la direction métonymique élaborée par les romanciers, au sens où l'histoire individuelle féminine s'emboîte dans l'histoire nationale, et mondiale par le fait des destins migratoires des personnages» (p. 45).

La seconda parte del saggio, «Fantaisies», tocca nel profondo la creazione dell'opera djebariana, indagando tutti quei sottili meccanismi dell'ars scribendi che portano il lettore a dire: «Ça, c'est du Djebar». Il primo capitolo «Fantaisies picturales» si concentra sull'influenza della pittura sulla scrittura. In particolare, Chikhi analizza come l'autrice abbia intessuto, pagina dopo pagina, un fitto dialogo con grandi pittori, e come la sua penna, quasi un "pinceau-plume», sia riuscita a descrivere narrativamente l'arte del movimento. Basti pensare alla raccolta Femmes d'Alger dans leur appartement, in cui si moltiplicano i riferimenti a Délacroix e a Picasso, definendo le tappe del percorso di liberazione delle donne d'Algeri. Nel secondo capitolo, l'attenzione si sposta su un'altra fonte d'ispirazione essenziale per l'autrice: la musica. Passando attraverso ritmi, trance, fughe e silenzi, Chikhi mostra gli aspetti musicali dell'opera di Assia Djebar, che applicano al testo scritto - testo-strumento secondo Chikhi - le modulazioni di un'opera musicale, dalla polifonia delle linee narrative all'importanza del tempo, «allegretto», «moderato», a volte "presto». La terza parte, «Réflexions et méditations» ci guida nella cartografia dei riferimenti storici e intertestuali, i cui punti cardinali sono dettati dalle Mille e una notte in Ombre sultane, dalle donne rawiyates, les transmetteuses della vita del Profeta, in Loin de Médine, fino a raggiungere la struttura di un complesso palinsesto storico ne Les Nuits de Strasbourg.

4 La quarta ed ultima parte, «Les territoires de la langue», si concentra sui territori (al plurale) della lingua. L'universo linguistico multiplo di un'Algeria che è da sempre crogiolo di culture e idiomi, con i suoi territori letterari marcati da plurilinguismo, se non da una forte diglossia, è terreno fertile per l'autrice che ha saputo sfruttarne, non senza un certo tiraillement, tutte le sfaccettature. L'opera di Assia Djebar è per Beida Chikhi un «texte-corps», corpo multiplo poiché «les langues se côtoient, se partagent les significations, se relaient, se soutiennent, se mêlent dans un corps 'polygame', se fécondent et se souviennent les unes des autres, comme dans une solidarité féminine, transmise par-delà les siècles» (p. 127).

5 In questo viaggio nella geografia reale e immaginaria di più paesi e di più lingue, anche l'esilio acquista una dimensione positiva. Come afferma Chikhi in conclusione, «d'une créativité à l'autre, la pensée de l'exil elle-même s'épuise dans le rythme de la marche, dans le voyage, dans la navigation des langues, dans le croisement des imaginaires, dans la mobilité romanesque, dans la fiction vive et la fantaisie du quotidien» (p. 150). Così, l'immenso edificio dell'opera di Djebar, definita scrittrice-architetto da Chikhi, si dispiega sotto gli occhi del lettore, svelato nella sua struttura interna. Ne emerge il ritratto di una grande autrice, il cui geste scriptural è determinato non solo dall'ascolto 
del sé, ma anche dal desiderio di produrre un discorso letterario più ampio, che investa la contemporaneità e coinvolga un insieme di conoscenze, arti e discipline (la storia, la sociologia, la psicanalisi, la linguistica, il cinema, la pittura, la musica...). Non bisogna infatti dimenticare che Djebar è scrittrice, critica, cineasta, storica dell'arte, drammaturga, e che tutti questi elementi si compenetrano nell'insieme della sua opera.

6 Un ricco compendio di documenti va infine a completare questo prezioso volume e a farne l'unicità: un articolo dell'arabista Jacques Berque, apparso su «Le Nouvel Observateur» nel 1985, e due interventi pubblici di Djebar, tra cui segnaliamo il discorso d'investitura all'Académie Française del 22 giugno del 2006. La sezione delle «annexes» si chiude con un importante apparato iconografico, che non fa che esaltare la ricchezza dell'opera djebariana, mostrandone l'ispirazione, riverberandone i significati, creando infiniti circuiti di senso. Un saggio sulla Grande Dame della letteratura maghrebina, rigoroso, acuto, coinvolgente. 